UCRL-JC-128623 Rev 2

PREPRINT

\title{
Picosecond Nonradiative Processes in Neodymium-doped Crystals and Glasses: Mechanism for the Energy Gap Law
}

\author{
C. Bibeau \\ S. A. Payne
}

This paper was prepared for submittal to the 13th Topical Meeting on Advanced Solid-State Lasers

Coeur d'Alene, ID

February 2-4, 1998

March 2, 1998

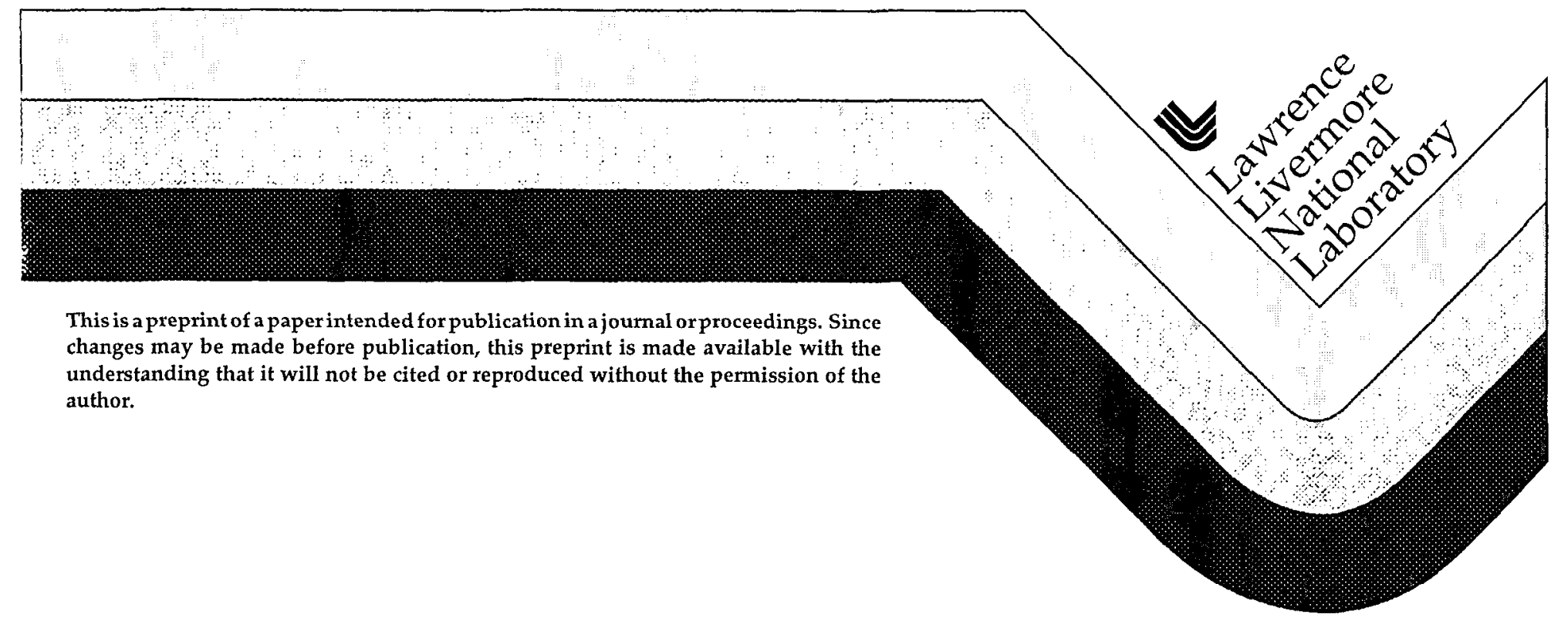




\section{DISCLAIMER}

This document was prepared as an account of work sponsored by an agency of the United States Government. Neither the United States Government nor the University of California nor any of their employees, makes any warranty, express or implied, or assumes any legal liability or responsibility for the accuracy, completeness, or usefulness of any information, apparatus, product, or process

disclosed, or represents that its use would not infringe privately owned rights. Reference herein to any specific commercial product, process, or service by trade name, trademark, manufacturer, or otherwise, does not necessarily constitute or imply its endorsement, recommendation, or favoring by the United States Government or the University of California. The views and opinions of authors expressed herein do not necessarily state or reflect those of the United States Government or the University of California, and shall not be used for advertising or product endorsement purposes. 


\section{Picosecond Nonradiative Processes in Neodymium-doped Crystals and Glasses: Mechanism for the Energy Gap Law*}

\author{
Camille Bibeau and Stephen A. Payne \\ University of California \\ Lawrence Livermore National Laboratory \\ P.O. Box 808 L-441 \\ Livermore, CA 94550 \\ Tel. (510) 422-7798, Fax (510) 423-6195
}

\begin{abstract}
We present measurements of the ${ }^{4} \mathrm{G}_{7 / 2}$ emission lifetime for $26 \mathrm{Nd}$-doped materials. A model of nonradiative decay based on dipole-dipole energy transfer is developed and found to be supported by our data.
\end{abstract}

\section{Introduction}

It has been extensively verified in the past that the nonradiative decay rate between rare earth energy levels is predominantly determined by the energy gap and the particular host medium. The energy gap law is embodied in the simple expression for the nonradiative rate:

$$
W_{n}=\left(1 / \tau_{0}\right) \exp (-a \cdot p),
$$

where $\tau_{0}$ and $\beta$ are constants characteristic of the host medium, and $p\left(=\Delta \mathrm{E} / \mathrm{h} v_{\max }\right)$ is the number of phonons needed to bridge the gap. The expression $h v_{\max }$ is related to the highest phonon frequency of the host medium and $\Delta \mathrm{E}$ is the energy gap betwcen the populated rare earth state and the next energetically lower level. The constant, $a$, is related to the details of the electron-phonon coupling. In nearly all cases rcported in the literature, eqn. (1) is regarded as adequately describing the measured decay rates over several orders of magnitude [1-3].

In the present paper we report the nonradiative decay rates of the ${ }^{4} \mathrm{G}_{7 / 2}$ state of $\mathrm{Nd}^{3+}$ in 26 different crystals and glasses, with the goal of providing a data base relevant to the relaxation rate of the ${ }^{4} \mathrm{I}_{1 / 2}$ state which has a similar energy gap as the ${ }^{4} \mathrm{G}_{7 / 2}$ state [4]. The ${ }^{4} \mathrm{I}_{11 / 2}$ state of $\mathrm{Nd}^{3+}$ is a particularly important one from a practical point of view, since it can potentially "bottleneck" during lasing and give rise to transient absorption at the laser wavelength (constituting loss).

Much of the motivation for establishing this correlation is that emission lifetimes are much simpler to measure and can be widely applied to numerous $\mathrm{Nd}$ doped crystals and glasses, while the pump-probe technique used to directly assess the ${ }^{4} \mathrm{I}_{11 / 2}$ decay time is complex to set up and execute.

The relevant energy levels are depicted in Fig. 1, where the $\tau_{5 / 2}, \tau_{7 / 2}$, and $\tau_{1 / 2}$ lifetimes are identified on the diagram along with the $532 \mathrm{~nm}$ pump wavelength and the $\sim 600 \mathrm{~nm}$ detection wavelength One of the details that must be handled in the numerical analysis of the data is the effect of the overlapping $\sim 600 \mathrm{~nm}$ emission arising from the ${ }^{4} \mathrm{G}_{5 / 2},{ }^{2} \mathrm{G}_{7 / 2}$ states on the measured results (i.e. $\tau_{5 / 2}$ ), as depicted in Fig. 1. The numerical analysis and resulting fits to the data will be discussed.

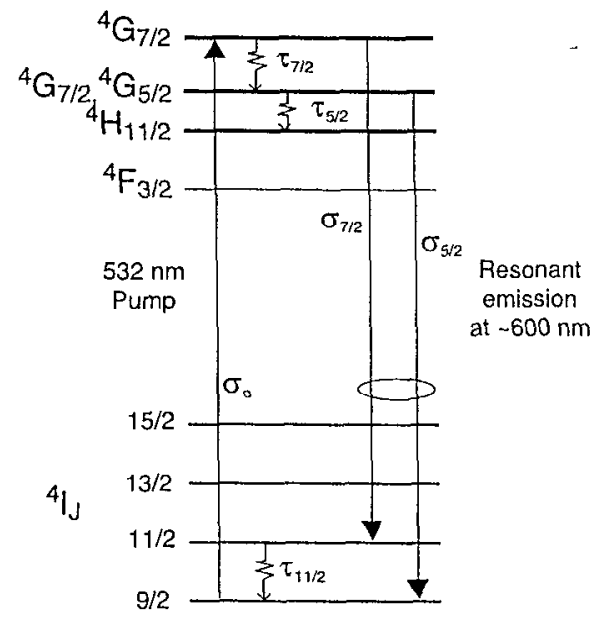

Fig.1 Energy levels and transitions for $\mathrm{Nd}^{3+}$

\section{Experimental}

The picosecond emission lifetimes [4] were measured using the time-correlated single photon counting system together with a Coherent mode-locked laser producing $\sim 90 \mathrm{psec}$ pulses at $76 \mathrm{MHz}$ and then doubled to $532 \mathrm{~nm}$. The sample emission was detected at $\sim 600 \mathrm{~nm}$ with a monochromator followed by a multichannel plate photomultiplier (MCP-PMT). The (deconvoluted) temporal resolution of the data is about $50 \mathrm{psec}$. For the case of the fluoride samples, the lifetimes of $\sim 10 \mathrm{nsec}$ were too long to be measured with the time-correlated photon-counting apparatus. To handle these longer lifetimes, we cmployed a conventional set-up consisting of a Q-switched

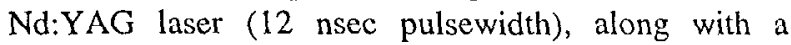
monochromator, oscilloscope, and MCP-PMT

\section{Results}

The results of the emission lifetime experiments are contained in Table 1. Based on an analysis of the best fit to the data, the uncertainty in the value of the $\tau_{7 / 2}$ lifetimes is $\Delta \tau_{7 / 2}(<1 \mathrm{~ns})= \pm 50$ ps for data with lifetimes less than $1 \mathrm{~ns}$ and $\Delta \tau_{7 / 2}(>1 \mathrm{~ns})= \pm 200 \mathrm{ps}$ for data with lifetimes greater than 1 ns.

Many of the trends that can be gleaned from the data in Table 1 are expected. For the case of the fluorides, the $\tau_{7 / 2}$ values are the longest, being 4,000 41,000 psec. This is anticipated, since their phonon 
frcquencies are the lowest among the hosts listed, being $<600 \mathrm{~cm}^{-1}$. Whereas the phosphate and silicate glasses are in the range of 150 to 250 psec owing to the rather high vibrational frequencies of the $\mathrm{SiO}_{4}$ and $\mathrm{PO}_{4}$ anions. It is noteworthy that comparisons of previous $\tau_{7 / 2}$ measurements in the literature are satisfactory, including [5]: 1400 versus 1090 psec for $\mathrm{YAlO}_{3} ; 370$ versus $200 \mathrm{psec}$ for $\mathrm{Y}_{3} \mathrm{Al}_{5} \mathrm{O}_{12}$; and 8400 psec versus 9100 psec for $\mathrm{LiYF}_{4} ; 56,400$ psec versus 41,000 psec for $\mathrm{LaF}_{3}$

Table 1. Emission lifetimes of the ${ }^{4} \mathrm{G}_{7 / 2}$ excited state $\left(\tau_{7 / 2}\right)$

\begin{tabular}{|c|c|c|}
\hline Name & Formula & $\tau_{7 / 2}$, psec \\
\hline $\begin{array}{l}\text { Phosphate } \\
\text { glasses }\end{array}$ & $\begin{array}{l}\mathrm{P}_{2} \mathrm{O}_{5}+\mathrm{Al}_{2} \mathrm{O}_{3}+ \\
\text { modifiers }\end{array}$ & \\
\hline LG-750 & & 228 \\
\hline APG-1 & & 215 \\
\hline APG- $x$ & & 210 \\
\hline APG -2 & & 150 \\
\hline LG-812 & $\begin{array}{l}\mathrm{P}_{2} \mathrm{O}_{5}+\text { fluoridest } \\
\text { modifiers }\end{array}$ & $\sim 1400$ \\
\hline $\begin{array}{l}\text { Silicate } \\
\text { glasses }\end{array}$ & $\begin{array}{l}\mathrm{SiO}_{2}+\mathrm{Al}_{2} \mathrm{O}_{3}+ \\
\text { modifiers }\end{array}$ & \\
\hline LG-660 & & 215 \\
\hline LG-650 & & 210 \\
\hline Sol-gel & $\mathrm{SiO}_{2}$ & 245 \\
\hline \multicolumn{3}{|l|}{ Vanadate } \\
\hline $\begin{array}{l}\mathrm{YVO}_{4} \\
\text { Tungstate }\end{array}$ & $\mathrm{YVO}_{4}$ & 190 \\
\hline \multicolumn{3}{|l|}{ Oxide } \\
\hline YALO & $\mathrm{YAlO}_{3}$ & 1090 \\
\hline GSGG & $\mathrm{Gd}_{3} \mathrm{Sc}_{2} \mathrm{Ga}_{3} \mathrm{O}_{12}$ & 715 \\
\hline YAG & $\mathrm{Y}_{3} \mathrm{Al}_{5} \mathrm{O}_{12}$ & 200 \\
\hline GGG & $\mathrm{Gd}_{3} \mathrm{Ga}_{5} \mathrm{O}_{12}$ & 530 \\
\hline LLGG & $\mathrm{La}_{3} \mathrm{Lu}_{2} \mathrm{Ga}_{3} \mathrm{O}_{12}$ & 1200 \\
\hline \multicolumn{3}{|l|}{ Apatites } \\
\hline C-FAP & $\mathrm{Ca}_{5}\left(\mathrm{PO}_{4}\right)_{3} \mathrm{~F}$ & 70 \\
\hline S-FAP & $\mathrm{Sr}_{5}\left(\mathrm{PO}_{4}\right)_{3} \mathrm{~F}$ & 175 \\
\hline C-VAP & $\mathrm{Ca}_{5}\left(\mathrm{VO}_{4}\right)_{3} \mathrm{~F}$ & 200 \\
\hline S-VAP & $\mathrm{Sr}_{5}\left(\mathrm{VO}_{4}\right)_{3} \mathrm{~F}$ & 330 \\
\hline ---.... & $\mathrm{Sr}_{5}\left(\mathrm{VO}_{4}\right)_{3} \mathrm{Cl}$ & 380 \\
\hline \multicolumn{3}{|l|}{ Fluorides } \\
\hline ZBLAN & $\begin{array}{l}\mathrm{ZrF}_{4}-\mathrm{BaF}_{2}-\mathrm{AlF}_{3}- \\
\mathrm{LaF}_{3}-\mathrm{NaF}\end{array}$ & 18,000 \\
\hline YLF & $\mathrm{LiYF}_{4}$ & 9100 \\
\hline $\mathrm{Na}_{3} \mathrm{Sc}_{2} \mathrm{Li}_{3} \mathrm{~F}_{12}$ & $\mathrm{Na}_{3} \mathrm{Sc}_{2} \mathrm{Li}_{3} \mathrm{~F}_{12}$ & 4000 \\
\hline $\mathrm{KY}_{3} \mathrm{~F}_{10}$ & $\mathrm{KY}_{3} \mathrm{~F}_{10}$ & 9000 \\
\hline $\mathrm{YF}_{3}$ & $\mathrm{YF}_{3}$ & 22,000 \\
\hline $\mathrm{LaF}_{3}$ & $\mathrm{LaF}_{3}$ & 41,000 \\
\hline
\end{tabular}

\section{Analysis and Discussion}

As mentioned earlier, measurements of the ${ }^{4} \mathrm{G}_{7 / 2}$ population decay time have long been suspected to be similar in magnitude to that of the ${ }^{4} \mathrm{I}_{11 / 2}$ level of $\mathrm{Nd}^{3+}$. Using the ${ }^{4} \mathrm{I}_{1 / 2}$ lifetimes from a direct measurement in [7] we find that the ${ }^{4} I_{1 / 2}$ and ${ }^{4} G_{7 / 2}$ lifetimes are correlated with each other to within about a factor of two, across a variety of different host media. This concurrence is reasonable, since some differences in the energy gap and perhaps the electron-phonon coupling may be expected, in addition to the experimental and analytical uncertainties. Furthermore, due to the small difference in the size of the encrgy gap for a given host medium, this data offers some experimental validation that the specific characteristics of the electronic states (symmetry, crystal field interactions, spin, etc.) do not strongly influence the nonradiative decay rate.

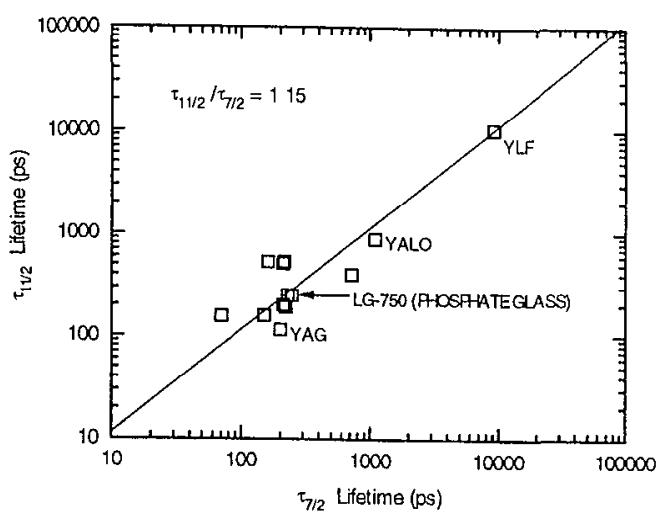

Fig. 2 Comparison of the ${ }^{4} \mathrm{I}_{11 / 2}$ nonradiative relaxation time from [4] and the ${ }^{4} \mathrm{G}_{7 / 2}$ emission lifetime.

Another theory of nonradiative decay derives the multi-phonon rate on basis of energy-transfer theory, as originally described by Forster [6] and Dexter [7]. With the assumption that dipole-dipole interactions dominate the process, and that the phonon absorption $\alpha_{\mathrm{pl}}(\lambda)$ is a slowly varying function compared to the emission cross section spectrum $\sigma_{\mathrm{em}}(\lambda)$, we derive that.

$$
\mathrm{W}_{\mathrm{nr}}=\frac{2 \mathrm{c} / 3}{(\pi \mathrm{n})^{2}} \frac{\int \sigma_{\mathrm{em}} \mathrm{d} \lambda}{\mathrm{V}_{\mathrm{min}}} \alpha_{\mathrm{ph}} \text {, }
$$

where $V_{\min }$ is the minimum volume that is nonabsorbing in nature (centered on the rare earth ion), $n$ is the refractive index, and $c$ is the speed of light. This expression essentially emerges from the well-known Forster-Dexter spectral overlap integral between the emitting and absorbing species, and a volume integration over a uniform acceptor (phonon) concentration, (a derivation similar to other reports in the literature [8-11]). It is also noteworthy however, that the multi-phonon absorption spectrum is commonly described with an exponential expression expression $[12,13]$

$$
\alpha_{\mathrm{ph}}=\mathrm{A}_{\text {host }} \exp \left(\begin{array}{ll}
-\gamma_{\text {host }} & \mathrm{h} \mathrm{v}_{\mathrm{ph}} / \mathrm{hv_{ \text {char } }}
\end{array}\right)
$$


where $v_{\text {ph }}$ is the phonon frequency, $v_{\text {char }}$ is a characteristic phonon frequency of the host material, and $\gamma_{\text {host }}$ and $A_{\text {host }}$ are other host-dependent constants. So if we combine eqns. (3) and (4), we can suggest an alternative route to deriving the form of the energy gap law.

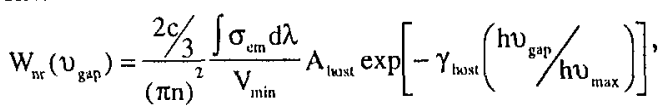

where we have identified $v_{\mathrm{ph}}$ as $v_{\text {gap }}$ and $v_{\text {char }}$ as $v_{\max }$ (maximum phonon frequency), the . The $\gamma_{\text {lost }}$ parameter turns-out to be similar for many crystals, for instance being in the range of 4-5 for alkali and alkaline-earth halide crystals [13]. The main point to note regarding eqn (4) is that the exponentiated factor in square brackets only contains information concerning the host medium, while the rare earth properties are exclusively represented in the pre-exponential factor - implying that we may expect reasonably good adherence to the form of the energy-gap law, eqn. (1),

We can explore the validity of eqn. (4) by inputting reasonable estimates for the terms in the preexponential factor and deducing the magnitude of this constant. Using $V_{\min }=5 \times 10^{-24} \mathrm{~cm}^{3}, A_{\text {host }}=20,000 \mathrm{~cm}^{\text {. }}$ ' (average from ref. [13]), and $\sigma_{\mathrm{em}} * \Delta \lambda=1.8 \times 10^{-25} \mathrm{~cm}^{2}$ (calculated from Judd-Ofelt theory using the average $\Omega_{\mathrm{J}}$ parameters of YAG and YLF [14J), we obtain a value of $0.6 \times 10^{12} \mathrm{sec}^{-1}$, or $\tau_{0}=1.7 \mathrm{psec}$, which is defined from eqn. (1) as:

$$
1 / \tau_{\mathrm{o}} \equiv \frac{2 \mathrm{c} / 3}{(\pi \mathrm{n})} \frac{\int \sigma_{\mathrm{em}} \mathrm{d} \lambda}{\mathrm{V}_{\min }} \mathrm{A}_{\mathrm{host}}
$$

We are now in a position to compare this calculation to the data in Table 1 , where we use the energy gap $\left(\Delta \mathrm{E}_{7 / 2}\right)$ and highest phonon frequencies $\left(h v_{\max }\right)$ to calculate number of phonons $p=\Delta E_{7 / 2} / h v_{\text {max }}$. If we also group all of the phosphate glasses into a single datum, and all of the silicates into a second one, then there are potentially ten usable points. Finally, if the $\mathrm{LaF}_{3}$ result is eliminated because of its strongly nonexponential character, the results of the exercise may be displayed as shown in Fig. 3, where we have plotted the nonradiative decay time, $\tau_{7 / 2}$, against the number of phonons, $p$. The data is then fitted to the reciprocal of the energy gap law:

$$
\tau_{\mathrm{nr}}=\tau_{0} \exp (\mathrm{a} \cdot \mathrm{p})
$$

with the result of the numerical fit yielding $\tau_{0}=3.3 \mathrm{psec}$ and $a=3.1$. We are very encouraged by this result because it may be compared with theoretical calculation of $\tau_{0}=1.7$ psec noted above in connection with eqn.
(5). Now, if we associate $\gamma_{\text {host }}$ with $a$, and $h v_{\text {char }}$ with $h v_{\max }$, the $a=3.1$ value from Fig. 3 appears to be within the range of what one may expect from the energytransfer theory of nonradiative decay encompassed in eqn. (4), since the phonon spectra of many materials is characterized by $\gamma_{\text {host }}=4-5$.

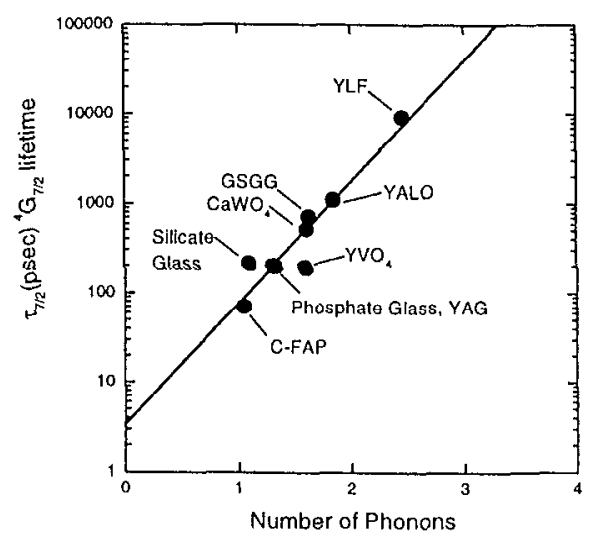

Fig. 3 Plot of the nonradiative decay time against the number of phonons nceded to bridge the gap.

In referring back to the more general energy-transferbased description of nonradiative decay relating to eqn. (2), we can apply another test of this model by plotting $\mathrm{W}_{\mathrm{m}}$ of the rare-earth ion against the absorption coefficient $\alpha_{\mathrm{pl}}$ of the host and fitting these data to the simple expression:

$$
\mathrm{W}_{\mathrm{nr}}=\mathrm{k} \alpha_{\mathrm{ph}} \text {, }
$$

where $\mathrm{k}$ can be theoretically calculated and related to $\tau_{0}$ by:

$$
\mathrm{k}=1 /\left(\mathrm{A}_{\text {host }} * \tau_{0}\right)=\frac{2 \mathrm{c} / 3}{(\pi \mathrm{n})^{2}} \frac{\int \sigma_{\mathrm{em}} \mathrm{d} \lambda}{\mathrm{V}_{\min }}
$$

based on eqns. (2) and (5). In Fig. 4 we have chosen to focus this analysis on data derived exclusively from the YAG, YALO and YLF hosts, because the phonon spectra of these materials have been reported in the literature $[15,16,17]$; in addition, the nonradiative decay rates and the associated energy gaps derived from refs. $[18,19,20,21,22]$ entail a wide variety of different electronic states and rare earth ions (in contrast to the prior exclusive attention given to the ${ }^{4} \mathrm{G}_{7 / 2}$ state of $\mathrm{Nd}^{3+}$ ). While we notice that the scatter in the data of Fig. 4 is substantial, it does span many orders of magnitude and the fitted or experimental value of $\mathrm{k}_{\text {exp }}=$ $1.0 \times 10^{7} \mathrm{~cm} / \mathrm{sec}$ based on the data is found to be near 
the independently theoretical value of $\mathrm{k}=3 \times 10^{7}$ $\mathrm{cm} / \mathrm{sec}$ deduced from eqn. (8) for the ${ }^{4} \mathrm{G}_{7 / 2}$ state.

Employing the experimental value of $k$ in the simple relationship of eqn. (8): $\tau_{0}=1 /\left(\mathrm{k}_{\text {exp }}{ }^{*} A_{\text {host }}\right)$, we find a third estimate for $\tau_{\mathrm{o}}$ of $5 \mathrm{psec}$, a value that is in reasonable accord with the theoretically derived value of $1.7 \mathrm{psec}$ from eqn. (5), and the other experimentally determined magnitude of $3.3 \mathrm{psec}$ from Fig. 3 and eqn. (6).

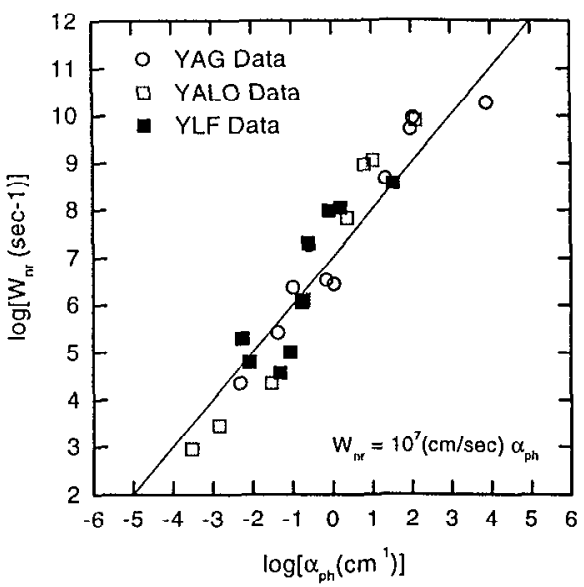

Fig 4. Plot of the nonradiative decay rate of various states of rare earth ions in YAG, YALO, and YLF, as a function of the phonon absorption coefficient of the host at the particular cnergy gap.

It is important to keep in mind that the results from the first two calculations are specific to the ${ }^{4} \mathrm{G}_{7 / 2}$ state, while the treatment in the last calculation entails numerous different rare earth ions and states (Fig. 4). The concurrence of these three independent approaches in estimating $\tau_{0}$ is remarkable and an implicit endorsement of the usefulness of the energy-transferbased mechanism of nonradiative decay.

\section{Acknowledgments}

We would like to thank Christopher Marshall, Gary Wilke, Howard Powell and Bill Krupke for their suggestions throughout this project.

*This work was performed under the auspices of the Division of Materials Science of the Office of Basic Energy Sciences, U. S. Department of Energy, and by the Lawrence Livermore National Laboratory under contract W-7405-ENG-48.

[1] H. W. Moos, J. Luminesc. 1/2 (1970) 106.

[2] W. D. Partlow and H. W. Moos, Phys. Rev. 157

(1967) 252.
[3] L. A. Riseberg, W. B. Gandrud, and H. W. Moos, Phys. Rev. 159 (1967) 262.

[4] C. Bibeau, Ph. D. Dissertation, "Evaluation of the ${ }^{4} \mathrm{I}_{11 / 2}$ terminal level lifetime for several neodymiumdoped laser crystals and glasses," April 25, 1995.

[5] T. T. Basiev, A. Yu. Dergachev, Yu. V Orlovskii, and A. M. Prokorov, J. Luminesc. 53 (1992) 19

[6] T. Forster, Z. Naturf. 49 (1949) 321.

[7] D. L. Dexter, J. Chem. Phys. 21 (1953) 836.

[8] N. T. Timofeev and E. B. Sveshnikova, Opt. Spectrosc. (USSR) 54 (1983) 595.

[9] V. L. Ermolaev and E. B. Sveshnikova, J. Luminesc. 20 (1979) 387.

[10] V. L. Ermolaev and E. B. Sveshnikova, Chem. Phys. Lett. 23 (1973) 349.

[11] E. B. Sveshnikova, N. T. Timofeev, and V. M. Zolotarev, Izvestiya Akademii Nauk SSSR. Scriya Fizicheskaya 44, (1980) 722.

[12] T. C. McGill, R. W. Hellwarth, M. Magir, and H. V. Winston, J. Phys. Chem. Solids 34 (1973) 2105.

[13] I. L. Boyer, J. A. Harrington, M. Haas, and H. B Rosenstock, Phys. Rev. B 11 (1975) 1665.

[14] R. P. Bauman and S. P. S. Porto, Phys. Rev 161 (1967) 842.

[15] V. P. Gaponsev, M. R. Sirtlanov, A. K. Gromov, A. A. Isineev, Proceedings on the International Conference on Lasers '81 (STS Press, McLean, 1981), p. 763.

[16] G. A. Slack, D. W. Oliver, R. M. Chrenko, and S. Roberts, Phys. Rev. 177 (1969) 1308.

[17] E. B. Sveshnikova, A. A. Stroganov, and N. T. Timofeev, Opt. Spectrosc. (USSR) 64 (1988) 43.

[18] M. J Weber, Phys. Rev. B 8 (1973) 54.

[19] A. M. Tkachuk, A. V. Khilko, and M. V. Petrov, Opt. Spectrosc. (USSR) 58 (1985) 216.

[20] T. 'T. Basiev, Yu. V. Orlovskii, K. K. Pukhov, V. B. Sigachev, M. E. Doroshenko, and I. N. Vorob'ev, J. Luminesc. 68 (1996) 241.

[21] G. M. Zverev, G. Ya. Kolodnyi, and A. M. Onishchenko, Sov. Phys. - JETP 33 (1971) 497.

[22] T. T. Basiev, A. Y. Dergachev, Yu. V. Orlovskii, and A. M Prokhorov, N. Vorob'ev, OSA Proceedings on Advanced Solid State Lasers 10 (1991) 358. 


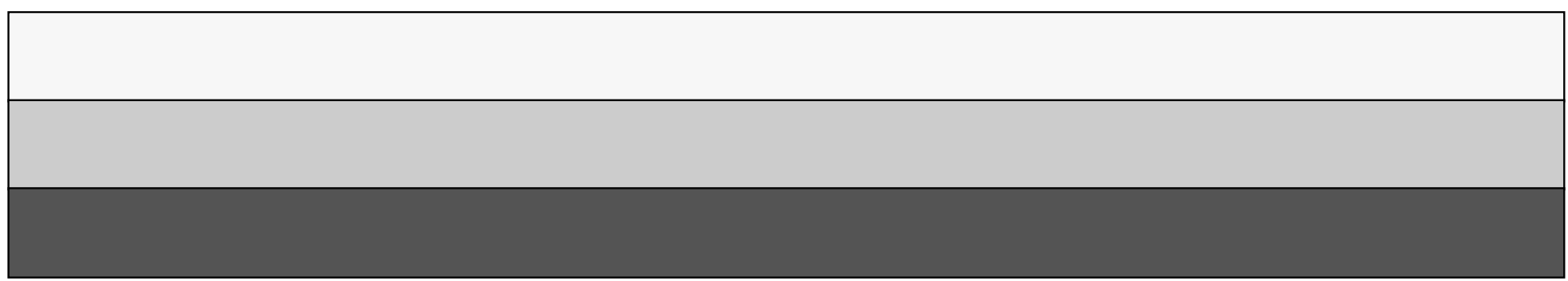

